\title{
Why is Drug-Induced Ototoxicity Still Not Preventable nor is it Treatable in 2019? - A Literature Review of Aminoglycoside and Cisplatin Ototoxicity
}

\section{Cherrabi Kaoutar*}

Department of Oto-Rhino-Laryngology and Cervico-Facial Studies, Fez, Morocco

${ }^{*}$ Corresponding author: Cherrabi Kaoutar, Resident, Department of Oto-Rhino-Laryngology and Cervico-Facial Studies, Fez, Morocco, Tel: 0679382957; E-mail: cherrabi.kaoutar@gmail.com

Received date: October 17, 2019; Accepted date: March 02, 2019; Published date: March 09, 2020

Citation: Cherrabi K (2020) Why is Drug-Induced Ototoxicity Still Not Preventable nor is it Treatable in 2019? - A Literature Review of Aminoglycoside and Cisplatin Ototoxicity. Arch Med Vol: 12 Iss: 2:3

Copyright: (C2020 Cherrabi K. This is an open-access article distributed under the terms of the Creative Commons Attribution License, which permits unrestricted use, distribution, and reproduction in any medium, provided the original author and source are credited.

\section{Abstract}

Oto-toxicity is defined by cochlear and or vestibular cellular lesions due to the use of drugs. The difference in specific mechanisms, the great semi logical variability, the heterogeneity of incriminated drugs, the inaccessibility to bimolecular and histological aspects in the current clinical practice, and the diverse aspects of post-lesional evolution: Are all factors that make it challenging to have systematic diagnostic protocols and systematic consensuses of management.

The possibility of replacement with less or non-toxic drugs relies on the peculiar understanding of mechanisms of action in a molecular level. Drugs with the same efficacious action and less or non-toxicity would offer a promising solution for this iatrogenic problem. For now: In most cases, these drugs are indispensable.

The idiosyncratic aspect of ototoxicity, the lack of consensus on screening and diagnostic protocols and classification systems, make it difficult to have an unbiased comparability regarding the different populations studied. The pure clinical and audiometric approach is syndromic, it evaluates lesions after their onset, and the classifications that are inspired from them have little specificity, sensibility and prognostic value in early stages.

The understanding of the different cellular modalities of inner ear drug-induced lesions related to each specific oto-toxic molecule, would allow: In the one hand: to establish a diagnostic test with high sensibility and specificity before the onset of irreversible cell damage. And in the other hand, would offer new insights into the possibilities of oto-protection, and specific treatment.

Oto-toxicity is a fertile field of scientific research which exploration has gained momentum in the last decade; in an experimental point of view, the bimolecular approach seems to be promising:
The thorough exploration of inner ear proteome, transcriptome, and genome in physiological and pathological contexts as potential biomarkers of early stages of cell lesions: The clinical application would not only allow a more specific understanding of different mechanisms of iatrogenic oto-toxicity, but also allow new insights into targeted protective and post-lesional therapies.

Keywords: Ototoxicity; Inner ear; Drug-induced deafness; Cisplatin; Aminoglycoside; Bimolecular; Screening proteome; Transcriptome; Heat shock protein

\section{Introduction}

"Primum non nocere", not to do harm is the first principle of medical practice. Drug-induced oto-toxicity has been mentioned in literature since the 1940s after the introduction of streptomycin [1-5].

It is a reversible or irreversible deficit induced by medication, prone to induce speech impairment especially in children, social intellectual and professional impairment, and low quality of life in adults. More than 746 molecules have been incriminated in oto-toxicity, especially those used in chemotherapy as cisplatin, and in severe infections as aminoglycosides. Knowledge about the molecular mechanisms of toxicity specific to every drug implicated is still unfolding, as well as the mechanisms of protection of inner ear structures. The systems that have been established often to classify ototoxicity meant to diagnose, and evaluate the gravity of ototoxicity when associated with the administration of chemotherapy protocols in clinical trials. [6-12]. The classification systems are mostly based on cochlear complications, with a few that mention vestibular disorders related to oto-toxicity. Significant gaps remain in our understanding of the molecular mechanisms underpinning auditory function, including the factors required for sensory cell regeneration.

This is a literature review about the fundamental knowledge concerning the clinical, physiological, and bimolecular aspect 
of oto-toxicity so far, in order to indicate potential diagnostic, preventive and therapeutic possibilities.

\section{Literature Review}

The goal of our study is to review the literature in order to allow a holistic view of ototoxicity, regarding the cellular and bimolecular, clinical, and therapeutic approach: In order to underline the current limitations in management of druginduced oto-toxicity. We surveyed the PubMed and Cochrane Databases.

\section{Objectives}

Ototoxicity: What do we know about physiological cellular and bimolecular aspects of oto-toxicity? What are yet the domains that are to be explored?

- Introduction to ototoxicity and the notion of benefice/risk balance.

- Basic anatomical and physiological notions.

- Homeostasis of inner ear.

- Molecular diffusion.

- Molecular targets and alteration in protein machinery.

- Mechanisms of protection.

- Role of inner ear immune system in ototoxic phenomena.

- Protein cascades, miRNA, and DNA loci implicated.

Ototoxicity: What do we know about ototoxicity management in clinical practice?

- Risk factors.

- Criteria of diagnosis.

- Classification: Diagnostic and prognostic.

- Oto-protection protocols.

\section{Results and Discussion}

We have used the keywords: Ototoxicity, inner ear, druginduced deafness, cisplatin, aminoglycoside, bimolecular, screening proteome, transcriptome, heat shock protein. We found 147824 articles, and after eliminating duplicated articles, and using filters (from January 1990 to October 2019, reviews).

The remaining articles were of 120 articles, stressing out several notions, after eliminating redundant information, 59 articles were kept.

In the one hand the clinical, audio logical and bimolecular aspects of ototoxicity, the classification, evaluation and treatment limitation encountered in clinical routine, and in the other hand the promises of the bimolecular approach of ototoxicity.

What do we know about physiological cellular and bimolecular aspects of oto-toxicity? What are yet the domains that are to be explored?

Introduction to ototoxicity and the notion of benefice risk balance: Oto-toxicity is an iatrogenic affection that is related to at least 746 molecules. It has been qualified as an adverse event for some anti-neoplastic treatment; however it is not labeled as a serious adverse event. Hence, in spite of life preserving benefits of these drugs, the possibility of permanent or significant disability must be considered and managed [1].

Drug research, bimolecular, genetic and proteomic understanding of cellular machinery at a molecular level are all fields that promise to clarify the actions that must be taken to insure an optimal targeted treatment with the less possible adverse effects. The study of inner ear physiology at this scale is a relatively new concept: this would not only allow a preventive approach to oto-toxicity, but would also open possibilities of early diagnosis, and targeted treatment.

Basic anatomy and physiology of inner ear: The inner ear is the sense organ of hearing and equilibrium, it has 6 sensory epithelia, five of which are related to the vestibule, and one concerns the cochlea: The organ of corti. The organ of corti contains 3 parallel tubes, scala tympani, scala vestibule and scala media.

Homeostasis of inner ear: The first step in understanding the phenomenon of oto-toxicity is to understand the basic physiology of fluid transport to inner ear environments, this implies understanding:

- Understanding the perilymph and endolymph homeostasis.

- Understanding the characteristics of the blood labyrinth barriers, and the mechanisms of entry of ototoxic drugs to the inner ear.

- Understanding the cellular and molecular impairment due to the presence of each oto-toxic drug.

Understanding the perilymph and endolymph homeostasis mechanisms of inner ear:

Perilymph and endolymph have a double role.

Mechanical role: Transmission of sound wave into the stereocilia of hair cells of inner ear, leading to activation of hair cells.

Electrical role: Provides ionic gradients that enhances sensitivity of hair cells, and contributes to signal transduction.

Endolymph and perilymph are different concerning the ionic and electrical characteristics.

Endolymph resembles intracellular environment: High concentration of potassium, and low concentration of sodium, in also contains calcium, proteins and glucose, with lower concentration compared to plasma.

The characteristics of endolymphatic sac are different from endolymph: It has inverted potassium and sodium concentration ratio like perilymph. But unlike endolymph and perilymph: It is more acidic environment.

Perilymph liquid is more similar to extracellular environment, characterized by high sodium concentration and low potassium concentration, it also contains chloride, protein and Glucose, exchanges between vestibular and tympanic environment occur in Helicotrema and spiral ligament. 
Endocochlear potential is the positive voltage of $80-100 \mathrm{mv}$, it is created by the difference in the ionic composition between endolymph and perilymph and it is generated and maintained by stria vascularis.

Endocochlear potential and potassium flux into hair cells are the main phenomenon that maintain the transduction of sound waves in hair cells, and enhances their sensitivity.

Potassium cycle in inner ear compartments: The main driving force of potassium is Endocochlear potential and resting membrane of hair cells.

The potassium channels play a very important role in generation of Endocochlear potential and potassium in intermediate cells of Stria Vascularis.

\section{Transport of molecules into the inner ear environment:}

The transport of substances inside inner ear involves several mechanisms: Diffusion, ultrafiltration, osmosis, lipid solubility, special tissue affinity, and the metabolism of inner ear tissues. The inner ear environments are protected from the general circulation by barriers; these are semi permeable membranes that are permeable only to certain molecules which criteria meet those of respective membrane $[3,4,9]$.

The space between the endolymph barrier and the perilymph barrier is called the vascular space.

Molecule diffusion: Characteristics of the Blood Labyrinth Barriers (BLB):

The blood labyrinth barriers consist on several inner ear membranes that separate different environments, they are meant to allow the selective passage of certain molecules; this selection depends on the nature of the membrane, and the molecules [2-4,10-15].

The basic structure of blood labyrinth is made of: Endothelial cells connected with tight junctions and resting on a basal membrane.

Lately, new cells have been described in the BLB: Pericytes and perivascular resident macrophages, they are known for regulating the barrier integrity, in their absence, the tight junctions are leaky to large molecules $[16,17]$.

Pericytes are rich in desmine, a protein that mechanical strength to capillaries and enhance general integrity of network. Pericytes and perivascular resident macrophage are new classes of cells in BLB, they are still largely uncharacterized.

In a molecular scale, the BLB contains a large number pf transporters and metabolic enzymes, which indicated a high level of energy and transport.

There are 5 types of blood labyrinth barriers:

- Blood/endolymph barrier: Located in Stria Vascularis

- Blood/perilymph barrier

- Cerebrospinal fluid/perilymph barrier

- Middle ear/labyrinth barrier (Round window)

- Endolymph/perilymph barrier

Molecule targets and proteins implicated in ototoxicity: In their path to hair cells, in the organ of corti, and the sensorial epithelium in the vestibule, ototoxic drugs ototoxic drugs are to go through blood labyrinth membranes [2-4,9,12,18-25].

At least 746 drugs have been identified ototoxic: Aminoglycoside, loop diuretics, non-steroid anti-inflammatory drugs and some anti-humoral drugs such as cisplatin. Each ototoxic drug has one or several targets inside the inner ear. The effect of all of them ultimately results in the loss of function of cochlea and/or vestibule at different severity scales. Aminoglycoside and cisplatin are mostly explored in the literature.

Mechanisms of ototoxicity of aminoglycosides: Streptomycin is the first aminoglycoside to be identified as an ototoxic drug. Several mechanisms of action have been mentioned.

Aminoglycosides are secreted completely unchanged by the kidney, the renal dysfunction increases the risk of ototoxicity. Genetic mutations of mitochondrial RNA have been associated with increased ototoxicity risk in several studies (Table 1).

Table 1 Labyrinthine barriers.

Labyrinthine barriers

Blood/ endolymph barrier: Located in Stria Vascularis
Only permeable to water molecules, ions such as $\mathrm{Cl}-, \mathrm{Na}+$ and $\mathrm{K}+$, besides oto-toxic drugs.

Made of tight junctions holding together marginal cells.

Considering oto-toxicity phenomenon's: It is at the same time a point of entry and a target of oto-toxic drugs, no exact mechanisms for either role, theories are stipulated.

Systemic gentamicin pathway across the stria Vascularis, only hair cell in the organ of Corti, and the marginal cells are affected by gentamicin [16].

The direct toxicity of gentamicin to Stria Vascularis is suspected

Studying molecular cascades implicated in the increased permeability of stria vascularis seems to be an interesting approach to understand the molecular protective therapies.

Example: $\mathrm{Na}+$ / K+-ATPase a1 (ATP1A1) abundantly found in the Stria Vascularis capillaries, interacts directly with a cascade that is directly implicated in the integrity of the blood labyrinthine barrier, it's interaction with protein kinase $\mathrm{C}$ and occludin due to noise increases its permeability [11]. 


\begin{tabular}{|c|c|}
\hline & The organ of corti depends on the stria vascularis for its function and survival $[12,13]$. \\
\hline \multirow{8}{*}{ Blood/ perilymph barrier } & $\begin{array}{l}\text { The spiral ligament, spiral limbus, modulus and osseous spiral lamina all contribute to the formation of the blood-perilymph } \\
\text { barrier [11,14]. Is similar to blood brain barrier }\end{array}$ \\
\hline & It conditions the diffusion to Scala Vestibuli. \\
\hline & Made of tight junctions of basal cells. Instead of one semipermeable membrane. \\
\hline & We don't have a database of the proteins of the perilymphatic barrier. \\
\hline & $\begin{array}{l}\text { Blood/perilymphatic barrier is permeable to osmotic drugs (mannitol, glycerine, and isosorbide), as well as to oto-toxic drugs } \\
\text { (Aminoglycosides, platinum based anti-tumor drugs and furosemide), and lipopolysaccharides. }\end{array}$ \\
\hline & The diffusion of gentamicin depends on the serum concentration. (H. Hahn) [14]. \\
\hline & $\begin{array}{l}\text { - In systemic application of gentamycin, the distribution in the scala tympani is apical to basal; while in local application the } \\
\text { distribution is basal to apical [14]. }\end{array}$ \\
\hline & Concentrations are less variable in systemic applications compared to local application. (H. Hahn) [14]. \\
\hline CSF/perilymph barrier & Through it, passage of a few ions, proteins and leukotrienes from CSF is allowed [4]. \\
\hline $\begin{array}{l}\text { Middle ear/ labyrinth barrier: } \\
\text { (Round window) }\end{array}$ & $\begin{array}{l}\text { Is the round window membrane (RWM): The middle ear-perilymphatic barrier is permeable to horseradish peroxidase, } \\
\text { cationic ferritin, as well as to } 1 \mu \mathrm{m} \text { latex spheres [4]. }\end{array}$ \\
\hline \multirow{2}{*}{ Endolymph/perilymph barrier. } & The perilymphatic-endolymph barrier has lower permeability than the middle ear/ perilymphatic barrier. \\
\hline & The endolymph, perilymph barrier allows water molecules, steroids, and does not allow oto-toxic drugs to pass through it. \\
\hline
\end{tabular}

\section{Three phases are described in the literature:}

First: Aminoglycoside are polycation and highly polar, electrostatic interaction of aminoglycoside with the plasmatic membrane results in the displacement of calcium and the reversible dysfunction of inner ear structure.

Second: The energy dependent uptake of aminoglycoside; possibly passive transport is associated.

Third: Intracellular drug action: AG interacts with PIP2 (phosphatidylinositol biphosphate) hydrolysis, this tempers with calcium stores mobilization, and thus with all cellular cascades involving calcium.

Ototoxicity implicates a higher permeability of LBL to certain molecules.

The inflammatory state caused by the infection, by release of endotoxin may contribute to increasing permeability and thus contribute to the risk of oto-toxicity. Other factors seem to contribute to potentiating cochlear uptake of AG: blood flow, active and passive functions of membrane related to channel membrane functions, as well as serum level of melatonin.

The main mechanism of action of aminoglycosides is the blocking of several transmembrane channels and membrane receptors, which enhances the drug uptake and accumulation inside the cell. Other mechanisms are explored: One of which is the endocytosis of AG into cytosol through lysosome-like vesicles, which release the drug once its capacity exceeded.

The ototoxicity of gentamycin is more vestibular than cochlear, it's the AG that is most studied regarding its ototoxic effect that is used in a therapeutic goal in Meniere's disease.

Cell death related to aminoglycoside oto-toxicity is related to different cell death pathways:
- Caspase dependent pathway.

- Caspase non-dependent pathway is mainly related to cathepsin.

- Other Jun-kinase inhibitors.

\section{Mechanisms of ototoxicity of cisplatin: [16-22]}

Several theories of cellular ototoxicity mechanisms have been reported:

- The activation of nicotinamide adenine dinucleotide phosphate oxidase 3 isoform (NOX3).

- Activation of transient receptor potential vanilloid (TRPV1 channel).

- The creation of reactive oxygen species, which involves activation of reactive oxygen species, depletion in glutathione that is a prominent antioxidant, the synthesis of enzymes, and an augmentation in the rate of lipid preoxidation.

Other theories are studies regarding ototoxicity mechanisms: The oxidative damage of proteins, nucleic acids damage by caspase system activation, and S-Nitrosylation of proteins in cochlear structures.

Targets of ototoxicity related to cisplatin are: outer hair cells, supporting cells, marginal cells of the stria vascularis, spiral ligament, and the spiral ganglion cells. The toxicity first targets basal hair cell, which results in an augmentation in high frequency thresholds in tonal audiometry, which evolves to upward until the deficit is complete. The lesion of the organ of corti is the most prominent.

Oto-protective mechanisms: The research of inner ear diseases, including oto-toxicity, promises to elucidate concepts about targeted inner ear therapies. Studying inner ear cellular lesional mechanisms in oto-toxic context has allowed to elucidate several therapeutic possibilities [20-25]. 
Oto-protective possibilities during lesional processes: Several physiological notions are explored in the experimental studies interested in the basics of oto-protective therapies.

Stria vascularis resembles the proximal tubules and Henle Loops of the kidney, both structures have Megalin: A vitamin $D$ transporter, the use of these channels is still being studied.

Targeting mechanotransducer channels: (MET channels) non-specific cation channels in hair cells: blockers like amiloride, curare, quinine, are toxic to human and can't be used as a therapeutic agent.

Calcium competitivity in MET channels: Calcium is considered to have a protective role in aminoglycoside ototoxicity. Deficit in MET channels seems to have a protective role in ototoxicity.

There are similarities in ribosome and bacterial ribosomal RNA, but there is proof of principle data that ototoxicity and antimicrobial effect related to aminoglycoside structure. Targeting alterative entry routes, through thorough study of structural subunit responsible for AG antibacterial role.

Endocytosis: The AG accumulates in lysosomes.

All oto-toxic drugs eventually results on formation of reaction oxygen species that trigger apoptosis.

Oto-protective therapies targeting endogenous otoprotective processes: Heat shock proteins are ubiquitous in all organisms. Also labeled chaperones, intrinsically generated proteins that protect inner ear structures from different extrinsic stressors [26-28]. They several roles in cellular machinery:

- Folding and unfolding of proteins.

- Transport and sorting into adapted cellular compartments.

- Antigenic presentation to complex of histocompatibility classes I and II.

- Cellular cycle and signaling protection.

Heat Shock Proteins (HSP), in the context of inner ear, is promising in discovering new approaches to efficacious protective therapies.

Transcriptional signature of HSP that protect against AG ototoxicity.

Induction of heat shock protein using heat shock stress can prevent both aminoglycoside and cisplatin induced ototoxicity in mice utricles in vitro.

Heat inducible from of HSP 70i.

Protection pharmaceutical: Inhibition of HSP 90, releasing transcriptional factor heart shock factor 1 (HSF1).

Other HSPs can be induced pharmaceutically (HSP27, HSP 40, HSP 70 ) reduce cisplatin oto-toxicity.

Related HSP: Hème oxygenase 1 (HO1 or HSP 32) protection in vitro and in vivo.

Identification of compounds that mimic heat shock have the potential to further oto-protective therapy to prevent hearing loss.
Innate inner ear immune system: The presence of immune cells inside the inner ear compartments is a subject that is yet to be explored and elucidated, although inflammatory reaction is thought to be contributing to inner ear insult, systemic modulation of this reaction could be a therapeutic possibility through enhancement of cascades contributing to otoprotection, for example: CX3CR1+ resident macrophages appear to regulate the influx of $\mathrm{CD} 45+$ cells after hair cell damage [28].

Role of inner ear immune system in ototoxicity: Infiltration of immune cells such as macrophages has been noted posterior to noise exposure and ototoxicity.

Toll-like receptors 4 (TLR4), cytokine, chemokine release and innate immune system infiltration, TLR4 activation, proinflammatory cytokine and chemokine release and infiltrating cells of the innate immune are results of injury to ototoxic effect of $A G$ and cisplatin. The understanding of the exact species of immune cells, their respective roles and the cascades is yet to be elucidated [28-32].

Role of adaptive immunity in the possibility of inner ear tissue regeneration is yet to be explored.

Proteome, transcriptome, and genome facts related to ototoxicity

Acute and chronic accumulation of damage to protein machinery of hair cells will damage to audition, by damaging the inner ear at one of these levels [33-38].

- Stereo cilia apparatus.

- Pre-synaptic signalling complex.

- Intracellular mechanisms for supporting highly metabolically active processes.

- Innate immune system.

The molecular bases of acquired hearing loss, due to noise and ototoxicity are poorly understood. Studying proteome, transcriptome and genome would allow thorough mapping of omics network, in physiological and pathophysiological ototoxic processes, and hence open horizons to non-invasive diagnostic tools, as well as oto-protective and targeted therapy.

Proteome: The composition of human perilymph and endolymph can be correlated to changes in function of inner ear [33-35].

The proteome plays an essential role in specialized hair cell function. Thousands of proteins are expressed in hair cells; hundreds of them are uniquely expressed in hair cells. Every ototoxic drug can have several targets on the inner ear apparatus, and every molecule has different targets and diffusion mechanisms.

A comprehensive data base of proteomic studies of structures of inner ear in physiological states is the first step in establishing the key proteins involved in key cascades to auditory function. A comparison of proteome can be made between proteome in healthy and pathological state can allow establishing correlations and drawing theories related to the role of proteins in the different cascades. 
Transcriptomes: Transcriptome analysis of single cells and purified specific inner ear hair cells gives a precise approach of developmental processes and essential gene expression of hair cells. miRNAs are prominent factors for post-transcriptional processes, as they controls gene expression at mRNA level [36-39].

In the human ear: miRNA fluid profile is unique and distinct from serum miRNA profile, miRNAs may serve as biomarkers. Databases of inner ear transcriptome have been established, and there are differences between cochlear inner ear cells and vestibular inner ear cells.

miRNA-205 serum level is cited as a diagnostic biomarker for aminoglycoside ototoxicity in mice treated with aminoglycoside antibiotics, no clinical studies have been found in human $[40,41]$. miR-183 cluster was found to be essential for the regeneration of hair cells after ototoxic insult in zebrafish larvae which makes it a new target for stimulation for hair cells regeneration.

The study of miRNA is a sensitive indicator of gene expression, whilst proteomic analysis allows the study of gene product maturation, and the measurement of functioning pathways. The levels of miRNA and proteins do not strictly correlate: Due to the presence of splices

Genome: Several mutations have been linked to predisposition to ototoxicity to aminoglycoside and cisplatin. Only two studies have evaluated polygenic effects on the vulnerability to cisplatin ototoxicity.

Oldenburg et al. evaluated the cumulated risks of combinations in variants of GSTT1, GSTM1 and GSTP1 [42-46].

- Pussegoda et al. also performed plurigenic analyses but included more complex models incorporating clinical and genetic variables. These studies point out the need to multigenic screens in assessment of risk for oto-toxicity, since the latter may be under-estimated when considering one marker.

- DNA mutations in the $12 \mathrm{~S}$ rRNA gene, such as m.1555A>G and m.1494C $>\mathrm{T}$, are more prone to AmAn-induced ototoxicity.

\section{Ototoxicity: What do we know about} ototoxicity management in clinical practice? What are the limitations?

\section{Risk factors of oto-toxicity}

Several risk factors are incriminated in the onset of ototoxicity:

Male gender: There were no associations between male gender and cisplatin ototoxicity, however new studies have established gender as an independent factor in cisplatin ototoxicity [47-49]. 3 reports of male gender association with as a predictive factor of ototoxicity, and suspected gender related predisposition and sensitivity.
Yancey et al. finds that the incidence of ototoxicity is four times more important in male population, in pediatric population.

Age: Young patients ( $<5$ year old), and older patients ( $<49$ years old) are more predisposed to ototoxicity.

Mutations: Rapid, irreversible hearing loss can happen in patients with genetic predisposition.

Inherited mutation in the mitochondrial $12 \mathrm{~S}$ ribosomal RNA gene that predisposes carriers to aminoglycoside ototoxicity. Up to $1 / 3$ of patients with aminoglycoside ototoxicity carry this mutation. The gene MT-RNR1 is a hotspot for this mutation. Two other mutations in the same mitochondrial gene affect a small minority of additional patients' diagnosis is possible in many cases.

Mutation of Megalin, Glutathione, and S-transferases gene polymorphism, influence cisplatin oto-toxicity.

No biomarkers are now used in clinical practice for ototoxicity diagnosis.

It is the challenge of genomic medicine to translate more than a decade-old knowledge into clinical practice.

\section{Cumulative dose}

- Cisplatin toxicity is related to cumulative dose.

- Gentamycin toxicity is related to single dose.

\section{Diagnostic of Ototoxicity}

The diagnosis of drug induced oto-toxicity is based on medical history, clinical symptoms, and tonal audiometry in congruence with other tests that depend on the situation. Obtaining a pre-treatment baseline audiometry for all patients is difficult in current practice [25,34,50-53].

Late onset of symptoms may delay the detection of deficit in its early stages; the belated diagnosis is related to the risk of irreversible deficit.

\section{Medical history: Searching for risk factors.}

Tonal audiometry is the test of choice, in order to diagnose, and classify oto-toxicity: examining absolute threshold or comparing thresholds of recurrent tonal audiometry to a base line test prior to exposition.

High frequency threshold audiometry has the best sensibility in diagnosing early onset of oto-toxicity.

Other test could accompany the diagnostic protocol (TEEOE, DPOEAE).

\section{Classifications}

Absence of an international consensus regarding classification system of oto-toxicity.

The classification systems respond to one of three objectives:

1. The early diagnosis of cochlear deficit after administration of ototoxic drugs (Screening). 
2. The monitoring of the evolution of lesions (Evaluation).

3. The assessment of the severity of lesions (Prognosis).

The categories are established according to audiological findings. No classification is established upon vestibular deficit.

The criteria are based on audiological changes concerning changes in hearing levels in high frequencies. There are three kinds of classifications:

- Classifications based on absolute thresholds.

- $\quad$ Comparison of thresholds after treatment to a baseline audiogram. (Significance of change in value).

- Or both.

The 13 classification systems specific to the evaluation of cochlear deficit in ototoxicity are cited in a systematic review by Crundwell et al.

The difference that is between the classification based on audiometric findings and the subjective auditory impairment reported by the patient shouldn't be ignored. Most are targeting pediatric patients as an assessment tool at the end of the clinical trial. Classification systems that aim to summarize the audiological findings for other specialists aren' $t$ of prognostic value.

\section{Oto-protection protocols}

Oto-protective drugs are substances that can reduce the ototoxic effects of ototoxic drugs, concomitant administration of ototoxic and protective drugs is an interesting approach to the studies that have been made in this subject are limited $[54,55]$.

There are ethical imperatives related to the administration of oto-protective drugs:

- No medical interaction leading to decrease of therapeutic effect of ototoxic drugs.

- The ototoxicity must be severe enough to motivate otoprotection protocol.

- No side-effect.

- Cost effective.

\section{Some drugs are FDA approved for oto-toxicity:}

- Sodium thiosulfate- Reduces cisplatin-induced toxicity by reducing Reactive Oxygen Species (ROS).

- N-acetylcysteine- Double anti-oxidative activity. Being a thiol, NAC acts as an anti-oxidant, being a precursor for Lcysteine promoting the endogenous anti-oxidant system.

- D-Methionine- is a precursor of L-methionine and has been proved to be an important protectant against cisplatininduced ototoxicity, is an antioxidant, protect the endogenous antioxidant system in the body

- Ebselen- a seleno-organic compound, acts as an anti-oxidant, and provides protection against cisplatin induced ototoxicity.

- Dexamethasone- through several mechanisms, downregulating cytokines, and upregulating anti-oxidant enzymes.

- Flunarizine- T-type-calcium channel antagonist.
- Lipoic acid, Aspirin- Were found to be oto-protective in animal models, however the effect were not identifiable in clinical context.

- Vitamin E, Gingo Biloba extract (GPE 761) and Statin drugs are still investigated in clinical studies. Several drugs are still studied in pre-clinical phase.

\section{Conclusion}

Ototoxicity is an iatrogenic affliction that is related to drugs that are mostly indispensable. The actual approach of this disease is limited due to the heterogeneous clinical aspect of this disease, and the persisting gaps in the different cascades implicated in inner ear homeostasis. The bimolecular approach of inner ear is a promising field of research that will allow great insights into inner ear targeted biomarkers and therapies, through a more accurate understanding of cellular machinery of inner ear physiology and pathophysiology, however the transfer of knowledge from an experimental point of view to clinical practice is difficult due to limitations related to ethical questions related to difficult access to inner ear studies without invasive sampling, safety, cost-effectively and pertinence of oto-protective and regenerative treatment in humans. However, a thorough understanding of the underlying cellular machinery is the first step to highly specificity and sensitivity screening of subclinical lesions, as well as a precise targeted protective and regenerative therapy of inner ear insults.

\section{References}

1. Khoza-Shangase K (2017) Risk versus benefit: Who assesses this in the management of patients on ototoxic drugs?. J Pharm Bioallied Sci 9: 171-177.

2. Yorgason JG, Fayad JN, Kalinec F (2006) Understanding drug ototoxicity: Molecular insights for prevention and clinical management. Expert Opin Drug Saf 5: 383-399.

3. Yates GK (1995) Cochlear structure and function. In: Handbook of perception and cognition. Moore BCJ (ed.), Academic Press, San Diego, CA, USA. pp: 41-74.

4. Sun W, Wuqing $W$ (2015) Review advances in research on labyrinth membranous barriers. J Otol 10: 99-104.

5. Ferrary E, Couloigner V, Sterkers O (2007) Physiologie des liquides labyrinthiques. EMC-Oto-Rhino-Laryngologie 2: 1-7.

6. Nin F, Hibino $H$, Doi $K$, Suzuki $T$, Hisa $Y$, et al. (2008) The endocochlear potential depends on two $\mathrm{K}+$ diffusion potentials and an electrical barrier in the stria vascularis of the inner ear. Proc Natl Acad Sci USA 105: 1751-1756.

7. Takeuchi S, Ando M, Kakigi A (2000) Mechanism generating endocochlear potential: Role played by intermediate cells in stria vascularis. Biophys J 79: 2572-2582.

8. Schacht J (1986) Molecular mechanisms of drug-induced hearing loss. Hear Res 22:297-304.

9. Salt AN (2005) Pharmacokinetics of drug entry into cochlear fluids. Volta Rev 105: 277-298.

10. Yang Y, Dai M, Wilson TM, Omelchenko I, Klimek JE, et al. (2011) $\mathrm{Na}+/ \mathrm{K}+-A T P a s e ~ \alpha 1$ identified as an abundant protein in the 
blood-labyrinth barrier that plays an essential role in the barrier integrity. PLoS One 6:e16547.

11. Huth ME, Ricci AJ, Cheng AG (2011) Mechanisms of aminoglycoside ototoxicity and targets of hair cell protection. Int J Otolaryngol 2011.

12. Liu H, Li Y, Chen L, Zhang Q, Pan N, et al. (2016) Organ of Corti and Stria Vascularis: Is there an Interdependence for Survival? PLOS ONE 11.

13. Shi $X$ (2016) Pathophysiology of the cochlear intrastrial fluidblood barrier (review). Hear Res 338: 52-63.

14. Hahn $\mathrm{H}(2013)$ Gentamicin concentration gradients in scala tympani perilymph following systemic applications. Audiol Neurootol 18: 383-331.

15. Dai CF (2008) A systemic gentamicin pathway across the stria vascularis. Hear Res 235: 114-124.

16. Shi X (2011) Physiopathology of the cochlear microcirculation. Hear Res 282: 10-24.

17. Fischel-Ghodsian N (2005) Genetic factors in aminoglycoside toxicity. Pharmacogenomics 6: 27-36.

18. Paken J, Govender CD, Pillay M, Sewram V (2019) A review of cisplatin-associated ototoxicity. Semin Hear. 40: 108-121.

19. Yancey A, Harris MS, Egbelakin A, Gilbert J, Pisoni DB (2012) Risk factors for cisplatin-associated ototoxicity in pediatric oncology patients. Pediatr Blood Cancer 59: 144-148.

20. Ross CJ, Katzov-Eckert H, Dubé MP, Brooks B, Rassekh SR, et al. (2009) Genetic variants in TPMT and COMT are associated with hearing loss in children receiving cisplatin chemotherapy. Nat Genet 41:1345-1349.

21. Sluyter $S$ (2003) Alterations in the stria vascularis in relation to cisplatin ototoxicity and recovery. Hearing Res 185: 49-56.

22. Paken J, Govender CD, Pillay M, Sewram Semin V (2019) A review of cisplatin-associated ototoxicity. Hear 40: 108-121.

23. Low WK, Sylvia Kong WW, Michelle Tan GK (2010) Ototoxicity from combined cisplatin and radiation treatment: $A n$ in vitro study. Int J Otolaryngol 2010.

24. Igumnova V, Veidemane L, Vīksna A, Capligina V, Zole E, et al. (2019) The prevalence of mitochondrial mutations associated with aminoglycoside-induced deafness in ethnic Latvi population: The appraisal of the evidence. J Hum Genet 64: 199-206.

25. O'Sullivan ME, Perez A, Lin R, Sajjadi A, Ricci AJ, et al. (2017) Towards the prevention of aminoglycoside-related hearing loss. Front Cell Neurosci 11: 325.

26. Li Z, Srivastava P (2004) Heat-shock proteins. Current Protocols in Immunology. 58.

27. Gong SS, Yan Z (2002) Expression of heat shock protein 70 in the cochlea in experimental autoimmune inner ear disease. Ann Otol Rhinol Laryngol 111: 275-280.

28. Ryals B, Rubel E (1988) Hair cell regeneration after acoustic trauma in adult Coturnix quail. Science 240: 1774-1776.

29. Sher A, Gazzinelli RT, Oswald IP, Clerici M, Kullberg M, et al. (1992) Role of T-cell derived cytokines in the downregulation of immune responses in parasitic and retroviral infection. Immunol Rev 127: 183-204.
30. Wood MB, Zuo J (2017) The contribution of immune infiltrates to ototoxicity and cochlear hair cell loss. Front Cell Neurosci 11: 106

31. Barker N, Van Es JH, Kuipers J, Kujala P, Van den Born M, et al. (2007) Identification of stem cells in small intestine and colon by marker gene Lgr5. Nature 449: 1003-1007.

32. Mietto BS, Mostacada K, Martinez AMB (2015) Neurotrauma and inflammation: CNS and PNS Responses. Mediators of Inflammation 2015: 1-14.

33. Hu BH, Zhang C, Frye MD (2018) Immune cells and non-immune cells with immune function in mammalian cochleae. Hear Res 362: 14-24.

34. Schmitt HA, Pich A, Schröder A, Scheper V, Lilli G (2017) Proteome analysis of human perilymph using an intraoperative sampling method. J Proteome Res 16: 1911-1923.

35. Samson J, Richard S, Donald C (2009) Auditory proteomics: Methods, accomplishments and challenges. Brain Res 277: 24-36.

36. Hickox AE, Wong AC, Pak K, Strojny C, Ramirez M (2017) Global analysis of protein expression of inner ear hair cells. J Neurosci 37:1320-1339

37. Yizhar-Barnea O, Avraham KB (2017) Single cell analysis of the inner ear sensory organs. The International Journal of Developmental Biology 61: 205-213.

38. Mahmoudian-sani MR, Mehri-Ghahfarrokhi A, Ahmadinejad F, Hashemzadeh-Chaleshtori M, Saidijam M, et al. (2017) MicroRNAs: Effective elements in ear-related diseases and hearing loss. Eur Arch Otorhinolaryngol 274: 2373-2380.

39. Rohde M, Sinicina I, Horn A, Eichner N, Meister G, et al. (2018) MicroRNA profile of human endo-/perilymph. J Neurol 265: 26-28.

40. Kim CW, Han JH, Wu L, Choi JY (2018) microRNA-183 is essential for hair cell regeneration after neomycin injury in Zebrafish. Yonsei Med J 59: 141-147.

41. Ross CJD, Katzov-Eckert H, Dubé MP, Brooks B, Rassekh SR, et al. (2009) Genetic variants in TPMT and COMT are associated with hearing loss in children receiving cisplatin chemotherapy. Nat Genet 41: 1345-1349.

42. Tserga E, Nandwani T, Edvall NK, Bulla J, Patel P, et al. (2019) The genetic vulnerability to cisplatin ototoxicity: A systematic review. Scientific Reports 9.

43. Oldenburg J, Fossa SD, Ikdahl T (2008) Genetic variants associated with cisplatin-induced ototoxicity. Pharmacogenomics 9: 1521-1530.

44. Gao Z, Chen Y, Guan MX (2017) Mitochondrial DNA mutations associated with aminoglycoside induced ototoxicity. J Otol 12: 1-8.

45. Knight KR, Kraemer DF, Neuwelt EA (2005) Ototoxicity in children receiving platinum chemotherapy: Underestimating a commonly occurring toxicity that may influence academic and social development. J Clin Oncol 23: 8588-8596.

46. Dulon D, Mosnier I, Bouccara D (2012) Ototoxicité médicamenteuse. EMC-Oto-rhino-laryngologie 7: 1-12.

47. Cary R, Clarke S, Delic J (1997) Effect of combined exposure to noise and toxic substances - Critical review of the literature. Ann Occup Hyg 41: 455-465. 
48. Neuwelt EA, Brock $P$ (2010) Critical need for international consensus on ototoxicity assessment criteria. J Clin Oncol 28: 1630-1632.

49. Hahn H, Salt AN, Schumacher U, Plontke SK (2013) Gentamicin concentration gradients in scala tympani perilymph following systemic applications. Audiol Neurootol 18: 383-391.

50. Ganesan P, Schmiedge J, Manchaiah V, Swapna S, Dhandayutham S, et al. (2018) Ototoxicity: A challenge in diagnosis and treatment. J Audiol Otol 22: 59-68.

51. Young TH (2018) Inner ear test battery in guinea pig models - A review. Acta Otolaryngol 138: 519-529.
52. Crundwell G, Gomersall P, Baguley DM (2016) Ototoxicity: (cochleotoxicity) classifications: A review. Int J Audiol, 55: 65-74.

53. Ferreira PJ, Nuno BL, Leandro R, Diamantino $H$, Bruno $D$, et al. (2017) Cochleotoxicity monitoring protocol. Spanish Otolaryngology Act 69: 105-109.

54. Konrad-Martin D (2010) Evaluation of audiometric threshold shift criteria for ototoxicity monitoring. J Am Acad Audiol 21: 301-314.

55. Hazlitt RA, Min J, Zuo J (2018) Progress in the development of preventative drugs for cisplatin-induced hearing loss. J Med Chem 61: 5512-5524. 